\title{
Novel Design of Recursive Differentiator Based on Lattice Wave Digital Filter
}

\author{
Richa BARSAINYA, Tarun K. RAWAT \\ Division of Electronics and Communication, Netaji Subhas Institute of Technology, Sector-3, Dwarka, \\ 110078 New Delhi, India \\ richa.barsainya@gmail.com, tarundsp@gmail.com \\ Submitted May 12, 2016 / Accepted October 7, 2016
}

\begin{abstract}
In this paper, a novel design of third and fifth order differentiator based on lattice wave digital filter (LWDF), established on optimizing $L_{1}$-error approximation function using cuckoo search algorithm (CSA) is proposed. We present a novel realization of minimum multiplier differentiator using LWD structure leading to requirement of optimizing only $N$ coefficients for $N$ th order differentiator. The $\gamma$ coefficients of lattice wave digital differentiator (LWDD) are computed by minimizing the $L_{1}$-norm fitness function leading to a flat response. The superiority of the proposed LWDD is evident by comparing it with other differentiators mentioned in the literature. The magnitude response of the designed LWDD is found to be of high accuracy with flat response in a wide frequency range. The simulation and statistical results validates that the designed minimum multiplier LWDD circumvents the existing one in terms of minimum $a b$ solute magnitude error, mean relative error $(d B)$ and efficient structural realization, thereby making the proposed LWDD a promising approach to digital differentiator design.
\end{abstract}

\section{Keywords}

Lattice wave digital filter, digital differentiator, wideband, $L_{1}$-CSA, minimum multiplier

\section{Introduction}

In recent years, digital differentiators are widely applied in various fields of signal processing, image processing, biomedical engineering, radar engineering, control systems, etc. [1-4]. The design and realization of digital differentiator have emerged as an active area of research due to its wide range of applications. Digital differentiators are effectively used to compute the time-derivative of real time and/or stored signal, which necessitates their results to be of high accuracy and its structural realization to be robust. The frequency response of an ideal digital differentiator is given by

$$
H_{\mathrm{d}}(\omega)=\mathrm{j} \omega
$$

where $\mathrm{j}=\sqrt{-1}$ and $\omega \in[0, \pi]$ is the normalized frequency. An ideal differentiator has a constant phase response of $\frac{\pi}{2}(\approx 1.57 \mathrm{rad})$ over the entire Nyquist frequency range. In available literature, several methods have been extensively explored for designing and implementing a digital differentiator. Interpolation and approximation based techniques [5-8] and different optimization techniques are most prevalent approaches used for the designing purpose [9-16]. The optimization based design of digital differentiator can be described as an approximation problem which comprises of four steps. First, a desired ideal frequency response of the digital differentiator is defined. Second, selection of type of system (either FIR or IIR). Third, developing an optimality criterion to approximate the ideal response. Lastly, an application of an optimization method to compute the optimal system coefficients.

In this paper, instead of a direct form of FIR or IIR system, a new and improved class of IIR system is reported due to design constraints of IIR system. IIR systems suffer from stability problem due to its recursive nature, especially when quantization of signal and coefficient is applied. IIR systems also have limitations like sensitivity to wordlength and coefficient round-off errors which make their implementation tricky. Direct form structure of IIR system based $N$ th order digital differentiator requires $2 N+1$ multiplier coefficients. Due to involvement of large number of multiplications in the filter algorithm, hardware implementation incurs excessive area, delays and power consumption. Analyzing all these limitations and considering the requirement of competent design of the digital differentiator, mathematical modelling based on the lattice wave digital filter system is proposed. The lattice wave digital filter is a specific class of wave digital filter [17]. LWDF structures flaunt many fascinating properties such as low coefficient sensitivity and consequently the low accuracy requirements for register wordlength, higher dynamic range, higher overflow level, lower round-off noise, assurance of stability and good nonlinear properties under finite arithmetic conditions where the effects of rounding, truncation and overflow are present [17-19]. In the past, LWDF structures are used for realizing lowpass-highpass filter, bandpass-bandstop filter and Hilbert transformers [20-22]. Their resulting struc- 
tures are highly modular, less sensitive and found to have minimum hardware which make them suitable for signal processors and VLSI implementation.

In the literature, many researchers have proposed different wideband recursive differentiators by approximating the ideal differentiator response efficiently in a full Nyquist frequency range. The use of evolutionary algorithms in optimization of digital operators is an exponentially increasing field with usage of different optimization algorithms for obtaining improved models of digital differentiator. These intelligent algorithms are capable of providing optimal results by minimizing any multi-modal error objective functions in less computation time. Simulated annealing (SA) technique is used to optimize the differentiator designed using segment rule [12]. Genetic algorithm (GA) was practiced for designing of second order recursive differentiator and the designs were shown to perform well in terms of both magnitude and phase response [16]. A third order wideband differentiator using linear programming (LP) technique was introduced in [9]. The techniques SA, GA and Fletcher and Powell optimization were incorporated to optimize the interpolated coefficients of first and higher order differentiator [13]. Furthermore, the modified particle swarm optimization (PSO) algorithm based digital differentiator design was presented and compared with the designs of GA, variants of PSO and PSO-GA hybrid techniques [14]. Jain et al. proposed IIR differentiators using minimax and pole, zero, and constant optimization (MPZCO) methods [15]. The objective of these algorithms are to minimize the mean square error approximation by searching for an optimized set of numerator and denominator coefficients of IIR system. Direct form structure of $N$ th order IIR differentiator requires $2 N+1$ multiplier coefficients. It complicates the search as large computational complexity occur due to the increased number of variable to be optimized and the constraints that need to be incorporated to ensure stability of an IIR system. On the contrary, LWDF is completely characterized by a set of coefficients $(\gamma)$ that have excellent dynamic range and low wordlength requirements and only $N$ number of $\gamma$ coefficients are to be optimized for the design of $N$ th order differentiator.

In this paper, CSA technique aims to find the optimal coefficients for LWDD that closely match the ideal counterpart by minimizing the fitness function, that is, $L_{1}$-norm error. The $L_{1}$-norm based optimality criterion is employed due to its ability to produce the flattest response over wide range of frequency that approaches the ideal one [23]. Thus, in order to design digital differentiator with desired response, the $L_{1}$-norm is hybridized with the evolutionary algorithm to find the system coefficients.

In this paper, novelty lies in the fact that the wideband differentiator designed using LWDF system will incorporate all the advantageous properties of LWDF with utilization of minimum hardware and also competent results are gained by using optimization techniques. The success of this combination is validated by the results and comparisons performed in this work. The capability of global search and optimal

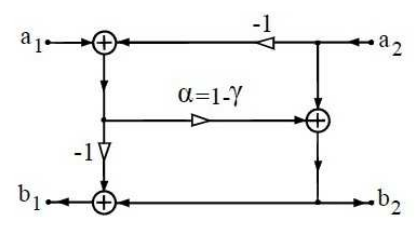

Type I: for $1 / 2<\gamma<1$

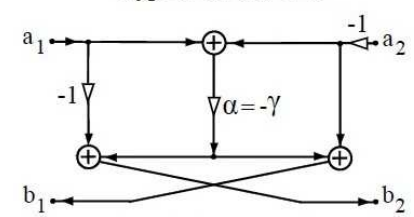

Type III: for $-1 / 2 \leq \gamma<0$

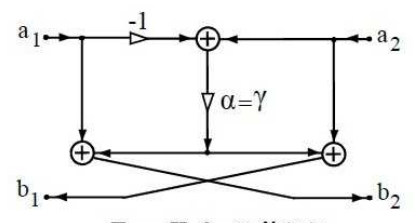

Type II: for $0<\gamma \leq 1 / 2$

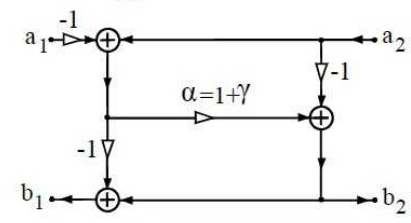

Type IV: for $-1<\gamma<-1 / 2$
Fig. 1. Signal flow graph of four symmetric two-port adaptors.

robust solution finding feature of cuckoo search algorithm is explored for the design of lattice wave digital differentiator. Thus, the hybrid $L_{1}-\mathrm{CSA}$ is formulated for designing of the LWDD in order to optimize $\gamma$ coefficients of a LWDF transfer function. The novel structural realization of the proposed 3rd and 5th order lattice wave digital differentiator is also introduced in this paper. The graphical analysis of magnitude response, phase response, magnitude error response and statistical analysis of absolute magnitude error (AME), mean relative error (MRE) in $\mathrm{dB}$ and mean phase error (MPE), demonstrates that the proposed minimum multiplier LWDD outperforms the reported differentiators.

The rest of the paper is segmented as follows. Brief overview of the LWDF along with the mathematical articulation is provided in Sec. 2. Section 3 focuses on the formulation of lattice wave digital differentiator design problem using $L_{1}$-error function. Section 4 provides the brief overview of the employed optimization algorithm. Simulation results and comparative analysis of the proposed LWDD is provided in Sec. 5 and Sec. 6. Section 7 highlights conclusion of the presented work.

\section{Lattice Wave Digital Filter}

This section gives a brief overview of lattice wave digital filter structure. Alike WDF, LWDFs are also related to certain analog prototype networks, i.e., lattice networks [17]. LWDF is represented by two parallel branches, which realize all-pass filters. These all-pass filters can be realized by using first- and/or second-order wave digital all-pass structures. These all-pass structures are implemented by using symmetric two-port adaptors and delay elements [18]. An adaptor requires a single multiplication and three additions. The application of adaptor produces an efficient realization in terms of number of multipliers for a given order and have low sensitivity to coefficient quantization. LWDF uses four types of adaptors as its building blocks. The signal flow graphs of four single multiplier symmetric two-port configurations are shown in Fig. 1.

According to [19], the adaptor coefficients $\gamma$ can be guaranteed to fall in the interval $-1<\gamma<1$. Methods to easily calculate these coefficients from the design specifica- 
tions have been discussed in [19]. The choice of an adaptor structure depends on the value of $\gamma$ coefficient. The $\alpha / \gamma$ conversion expressions is shown in Fig. 1. The value $\alpha$ coefficient should always be positive and less than or equal to half i.e., $0<\alpha \leq \frac{1}{2}$.

\section{Problem Formulation}

The ideal differentiator in (1) is approximated to the LWDF system. The transfer function of LWDF system is specified as

$$
H_{\mathrm{LWDF}}(z)=\frac{1}{a}\left[H_{1}(z)-H_{2}(z)\right]
$$

where $H_{1}(z)$ and $H_{2}(z)$ are allpass filter of order $P$ and $Q$, respectively. In case of lowpass/highpass filters, $P=Q-1$ or $P=Q+1$. The overall order of $H_{\mathrm{LWDF}}(z)$ is $P+Q . H_{1}(z)$ and $H_{2}(z)$ can be expressed in terms of adaptor coefficients as

$$
\begin{gathered}
H_{1}(z)=\frac{-\gamma_{0}+z^{-1}}{1-\gamma_{0} z^{-1}} \prod_{l=1}^{m} \frac{-\gamma_{2 l-1}+\gamma_{2 l}\left(\gamma_{2 l-1}-1\right) z^{-1}+z^{-2}}{1+\gamma_{2 l}\left(\gamma_{2 l-1}-1\right) z^{-1}-\gamma_{2 l-1} z^{-2}}, \\
H_{2}(z)=\prod_{l=m+1}^{m+n} \frac{-\gamma_{2 l-1}+\gamma_{2 l}\left(\gamma_{2 l-1}-1\right) z^{-1}+z^{-2}}{1+\gamma_{2 l}\left(\gamma_{2 l-1}-1\right) z^{-1}-\gamma_{2 l-1} z^{-2}}
\end{gathered}
$$

where $m=\frac{P-1}{2}$ and $n=\frac{Q}{2}$ and $\gamma$ is the adaptor coefficient that characterizes LWDF.

In this paper, design of $3 \mathrm{rd}$ and 5 th order differentiator is considered. The differentiator of even order can also be realized using LWDF, however, in that case complex coefficients are required. In this paper, only real coefficients, odd order differentiator design is considered and the transfer function of 3rd and 5th order LWDF system for designing of differentiator is expressed as

$$
\begin{aligned}
& H_{\mathrm{LWDF}}^{3 \mathrm{rd}}(z)= \\
& \frac{1}{a}\left[\left(\frac{-\gamma_{0}+z^{-1}}{1-\gamma_{0} z^{-1}}\right)-\left(\frac{-\gamma_{1}+\gamma_{2}\left(\gamma_{1}-1\right) z^{-1}+z^{-2}}{1+\gamma_{2}\left(\gamma_{1}-1\right) z^{-1}-\gamma_{1} z^{-2}}\right)\right], \\
& H_{\mathrm{LWDF}}^{\text {5th }}(z)= \\
& \frac{1}{a}\left[\left(\frac{-\gamma_{0}+z^{-1}}{1-\gamma_{0} z^{-1}}\right)\left(\frac{-\gamma_{1}+\gamma_{2}\left(\gamma_{1}-1\right) z^{-1}+z^{-2}}{1+\gamma_{2}\left(\gamma_{1}-1\right) z^{-1}-\gamma_{1} z^{-2}}\right)\right. \\
& \left.-\left(\frac{-\gamma_{3}+\gamma_{4}\left(\gamma_{3}-1\right) z^{-1}+z^{-2}}{1+\gamma_{4}\left(\gamma_{3}-1\right) z^{-1}-\gamma_{3} z^{-2}}\right)\right]
\end{aligned}
$$

where $a$ is the scaling factor. In order to obtain the digital differentiator with the desired specification, an objective function in terms of error between the ideal frequency response and LWDF frequency response is developed. The objective function is formulated using $L_{1}$-norm to obtain a set of optimized coefficients and can be expressed as

$$
\|E\|_{1}=\sum_{\omega}|\mathrm{e}(\omega)|
$$

where $\|$.$\| denotes norm of the function and \mathrm{e}(\omega)=H_{\mathrm{d}}(\omega)-$ $H_{\mathrm{LWDF}}(\omega)$ is the error objective function. The objective fitness function is minimized iteratively to obtain the optimized $\gamma$ coefficients of the 3rd and 5th order LWDF (given in (5) and (6), respectively) to design minimum multiplier differentiator with desired specifications. The motivation behind implementing the $L_{1}$-norm fitness function is due to the fact that it is capable of delivering flattest response amongst others, such as the $L_{2}$ and $L_{\infty}$-norms [23], [24].

\section{Brief Outline of Cuckoo Search Al- gorithm}

In this paper, CSA is used to design minimum multiplier digital differentiator. CSA tries to find the optimal $\gamma$ coefficients by iteratively minimizing the error objective function, leading to development of the lattice wave digital differentiator. In previous literatures, GA and PSO are most utilized optimization algorithm for designing of optimal recursive wideband differentiators. However, when tested on different benchmark functions, CSA found to be potentially more powerful than the GA and PSO [25].

Cuckoo search algorithm is instigated by the unique breeding behavior of cuckoo bird and the concept of Lévy flights which are observed in some species of birds and animals [25-27]. Cuckoo birds depend on some other bird's nest for hatching their eggs. Cuckoo attempts to determine a nest where the host bird has recently laid eggs. Cuckoo uses this nest to hide its own eggs. If the host bird identifies that the eggs are not its own, it may abandon the nest or either choose to destroy the alien eggs. This heads to the evolution of the cuckoo eggs, which make attempt to mimic the eggs of the host bird.

To apply cuckoo search algorithm, following assumptions are considered:

(i) Cuckoo bird hatches one egg at a time and hide it in the host's nest chosen randomly.

(ii) The nests with the best host environment and with the best eggs will survive and move forward to the next generations.

(iii) The number of host nests is fixed.

(iv) The probability of identifying alien eggs by the host bird is $P_{\mathrm{a}}$.

The Lévy flights are the forward steps taken by living beings, such as birds, insects and animals in search of their food. Lévy flight refers to a series of straight line flights followed by sudden $90^{\circ}$ turns. The usage of Lévy flight for choosing a random new nest is the main factor for improving the performance of CSA. To get a new random nest following Lévy flight based formula is used,

$$
x_{l}=x_{i}+v \oplus \operatorname{Lév} y(\lambda)
$$




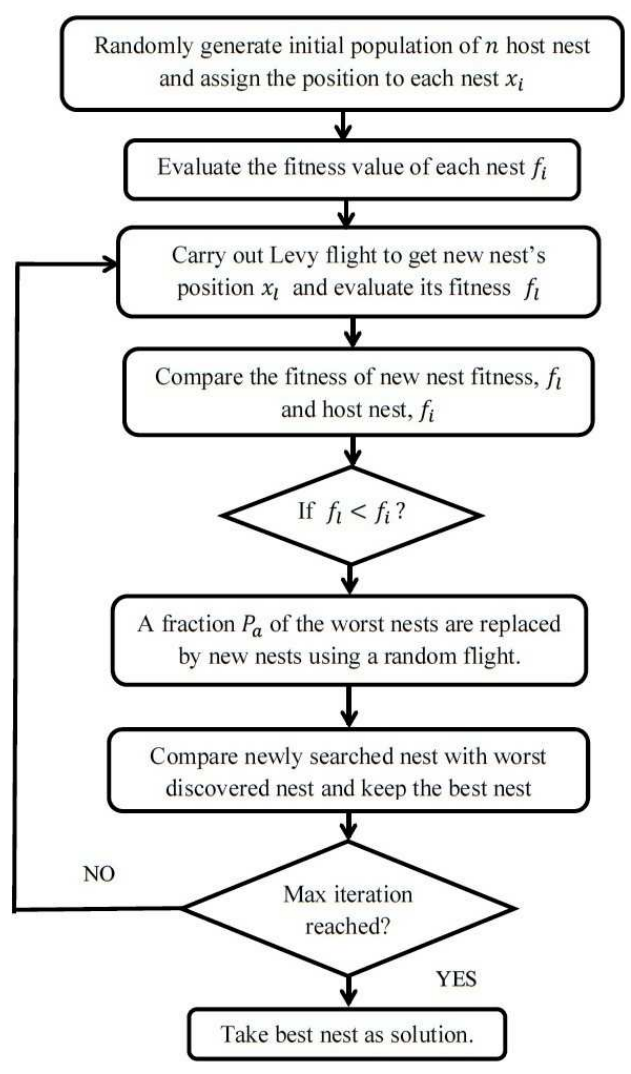

Fig. 2. Flow chart of Cuckoo search algorithm.

where $v$ is the step size related to the problem specified, $\oplus$ represents entry wise multiplication and $\lambda$ is a Lévy flight parameter. This equation represents a random walk which is a Markov chain which means its next step depends on the current location and the transition probability. The implementation steps of CSA are explained with a flow chart presented in Fig. 2.

\section{Simulation Analysis}

This section shows the design process of lattice wave digital differentiator using CSA and its efficient LWD structural realization with minimum multipliers. To incur the proposed LWDD, simulations are performed in MATLAB on Intel Core i5, $3.20 \mathrm{GHz}$ with $2 \mathrm{~GB}$ RAM. The optimal set of CSA parameters for design of minimum multiplier differentiator are reported in Tab. 1. After exhaustive simulation and analysis, the best optimal coefficients for the designed 3rd and 5th order LWDD are calculated by minimizing error fitness function using $L_{1}$-CSA are reported in Tab. 2 . Best results are reported here after 100 simulation trails with random parameter value selection. The tuning of controlling parameters is a typical task and there exists no definite methodology in the available literature to provide an optimal set of parameter values. So, optimal parameter values can differ for different problems.

\begin{tabular}{lc}
\hline Parameters & CSA \\
\hline Population size & 25 \\
Maximum iterations & 300 \\
Tolerance & $10^{-5}$ \\
Lower bound $\left(X_{\min }\right)$ & -1 \\
Upper bound $\left(X_{\max }\right)$ & 1 \\
Initial value of all coefficients & 0.01 \\
Discovering rate of alien eggs $\left(P_{\mathrm{a}}\right)$ & 0.25 \\
\hline
\end{tabular}

Tab. 1. Control Parameters of CSA for the optimized LWDD.

\begin{tabular}{ccccc}
\hline Order & $\begin{array}{c}\gamma \\
\text { coefficients }\end{array}$ & $\gamma$ range & $\begin{array}{c}\text { Adaptor } \\
\text { type }\end{array}$ & $\begin{array}{c}\alpha \\
\text { coefficients }\end{array}$ \\
\hline 3rd & $\gamma_{0}=-0.41434$ & $-\frac{1}{2} \leq \gamma<0$ & III & $\alpha_{0}=0.41434$ \\
& $\gamma_{1}=-0.06457$ & $-\frac{1}{2} \leq \gamma<0$ & III & $\alpha_{1}=0.06457$ \\
& $\gamma_{2}=-0.64613$ & $-1<\gamma<-\frac{1}{2}$ & IV & $\alpha_{2}=0.35387$ \\
& $a=0.65775$ & & & \\
\hline 5th & $\gamma_{0}=-0.08045$ & $-\frac{1}{2} \leq \gamma<0$ & III & $\alpha_{0}=0.08045$ \\
& $\gamma_{1}=-0.33818$ & $-\frac{1}{2} \leq \gamma<0$ & III & $\alpha_{1}=0.33818$ \\
& $\gamma_{2}=-0.91047$ & $-1<\gamma<-\frac{1}{2}$ & IV & $\alpha_{2}=0.08953$ \\
& $\gamma_{3}=-0.20044$ & $-\frac{1}{2} \leq \gamma<0$ & III & $\alpha_{3}=0.20044$ \\
& $\gamma_{4}=-0.83884$ & $-1<\gamma<-\frac{1}{2}$ & IV & $\alpha_{4}=0.16116$ \\
& $a=0.64531$ & & & \\
\hline
\end{tabular}

Tab. 2. Optimized Coefficients for the 3 rd and 5th order LWDD using $L_{1}$-CSA.

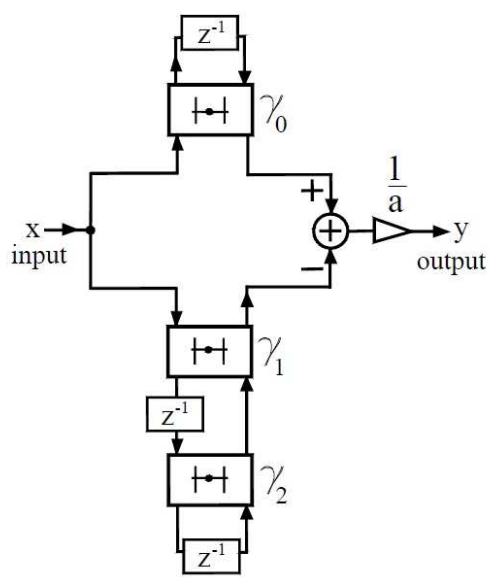

Fig. 3. Structural realization of the $3 \mathrm{rd}$ order lattice wave digital differentiator.

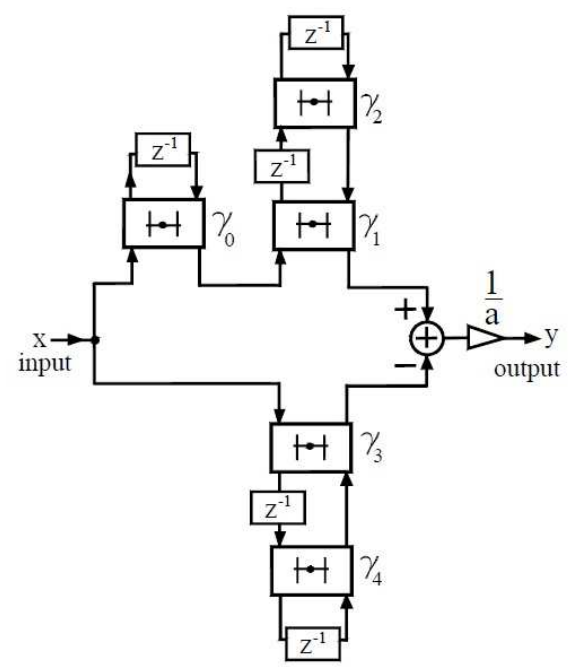

Fig. 4. Structural realization of the 5th order lattice wave digital differentiator. 


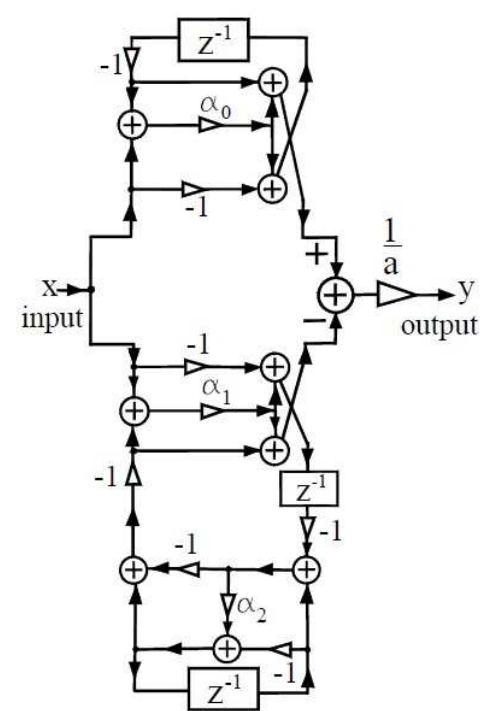

Fig. 5. Signal flow diagram of the 3rd order lattice wave digital differentiator.

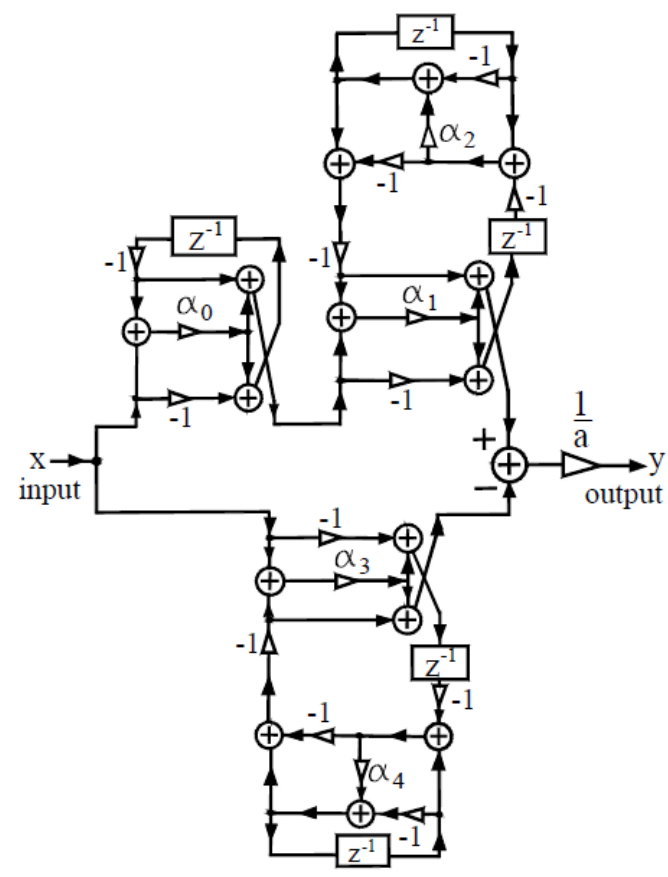

Fig. 6. Signal flow diagram of the 5 th order lattice wave digital differentiator

Table 2 also provides information about the adaptor type chosen depending on the value of $\gamma$ coefficients and the exact value of adaptor multiplier coefficients, $\alpha$. The lattice wave digital realization of the designed 3rd order LWDD is shown in Fig. 3. A minimum multiplier design for the 3rd order LWDD is procured where only three adaptors are utilized, where each adaptor comprises of a single multiplier. The lattice wave digital realization of the designed 5th order LWDD is shown in Fig. 4.

A minimum multiplier design for the 5th order LWDD is obtained, thereby reducing the computational requirement or the complexity of hardware in implementation. The signal flow diagram of 3rd and 5th order lattice wave digital differentiator is depicted in Figures 5 and 6, respectively. The absolute magnitude error of 1.4706 and 0.3243 have been achieved for the 3rd and 5th order LWDD optimized using $L_{1}$-CSA. The execution time to achieve corresponding values of AME and MPE is 80.4564 second and 106.2131 second, respectively. Furthermore, statistical analysis shows that the mean relative error $(\mathrm{dB})$ of -51.7828 and -64.0481 have been achieved for the 3rd and 5th order LWDD optimized using CSA. The MRE is computed over the complete frequency range using following equation

$$
M R E=\frac{1}{N} \sum_{i=1}^{N}\left|\frac{H_{\mathrm{d}}(\omega)-H_{\mathrm{LWDF}}(\omega)}{H_{\mathrm{d}}(\omega)}\right|, \quad 0 \leq \omega \leq \pi
$$

\section{Comparison of the Proposed Differ- entiators with the Existing Ones.}

To verify and evaluate the efficiency, the designed LWDD is compared with the existing differentiators. Several existing differentiators procured through different methods are tabulated in Tab. 3 for comparison.

The comparison is performed on the basis of procured magnitude response, magnitude error response and phase curve. The magnitude response plots of 3rd and 5th order LWDDs are obtained by simulating (5)-(6), after substituting the value of obtained optimal $\gamma$ coefficients. Figures 7 and 8 , represent the graphical comparison of magnitude and phase response of the 3rd order LWDD and all the existing 3rd order differentiators mentioned in Tab. 3, along with the ideal response. The magnitude error plot of the designed and reported 3rd order differentiators is shown in Fig. 9.

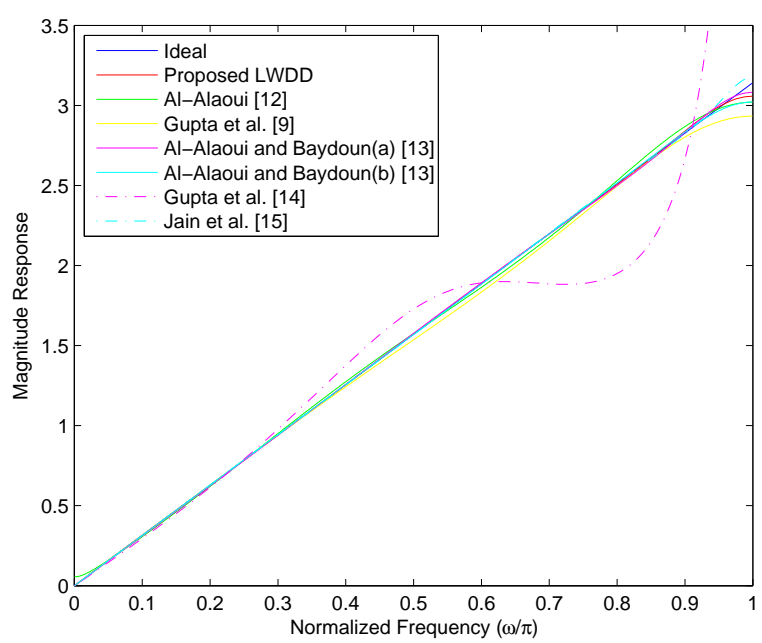

Fig. 7. Magnitude response comparison of the proposed 3rd order LWDD and existing differentiators. 


\begin{tabular}{clcll}
\hline Order & Reference & Method & $\begin{array}{l}\text { Numerator } \\
\text { coefficient }\end{array}$ & $\begin{array}{l}\text { Denominator } \\
\text { coefficient }\end{array}$ \\
\hline 3rd & Proposed LWDD & CSA & $0.53177 ; 0.8672 ;-0.8672 ;-0.53177$ & $1.0000 ; 1.1022 ; 0.34957 ; 0.02675$ \\
& Al-Alaoui & 3-segment & $0.01903 ;-0.02905 ; 1.1230 ;-1.1810$ & $1.0000 ; 0.1846 ;-0.001748 ; 0.03484$ \\
& Gupta et al. & LP & $1.0000 ;-1.0000 ; 0.0000 ; 0.0000$ & $0.3290 ; 0.8077 ;-0.1694 ; 0.0338$ \\
& Al-Alaoui and Baydoun (a) & GA & $1.1533 ;-0.4432 ;-0.7060 ;-0.0041$ & $1.0000 ; 0.7981 ; 0.0884 ; 0.0000$ \\
& Al-Alaoui and Baydoun (b) & SA & $1.1555 ;-0.3580 ;-0.7140 ;-0.0833$ & $1.0000 ; 0.8662 ; 0.1612 ; 0.0028$ \\
& Gupta et al. & PSO & $0.3237 ; 1.0000 ;-0.7133 ;-0.6124$ & $1.0000 ; 1.0000 ; 0.2759 ; 0.1595$ \\
& Jain et al. & MPZCO & $1.0000 ;-0.0243 ;-0.8315 ;-0.1436$ & $0.8646 ; 1.0001 ; 0.2470 ; 0.0056$ \\
\hline 5th & Proposed LWDD & CSA & $0.1295 ; 0.6828 ; 0.6019 ;-0.6019 ;-0.6828 ;-0.1295$ & $0.6541 ; 1.3175 ; 0.9691 ; 0.3156 ; 0.0435 ; 0.0018$ \\
& Devate et al. & curve fitting & $0.2500 ; 2.1408 ; 2.0522 ;-2.0523 ;-2.1407 ;-0.2499$ & $2.2257 ; 4.1683 ; 2.6300 ; 0.6353 ; 0.0517 ; 0.0000$ \\
\hline
\end{tabular}

Tab. 3. Optimal coefficients of the existing digital differentiators using different design methods.

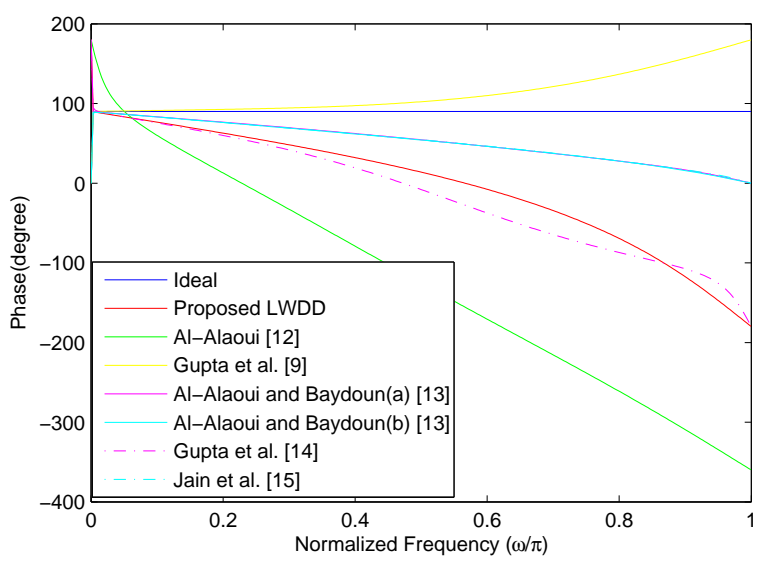

Fig. 8. Phase response comparison of the proposed 3rd order LWDD and existing differentiators.

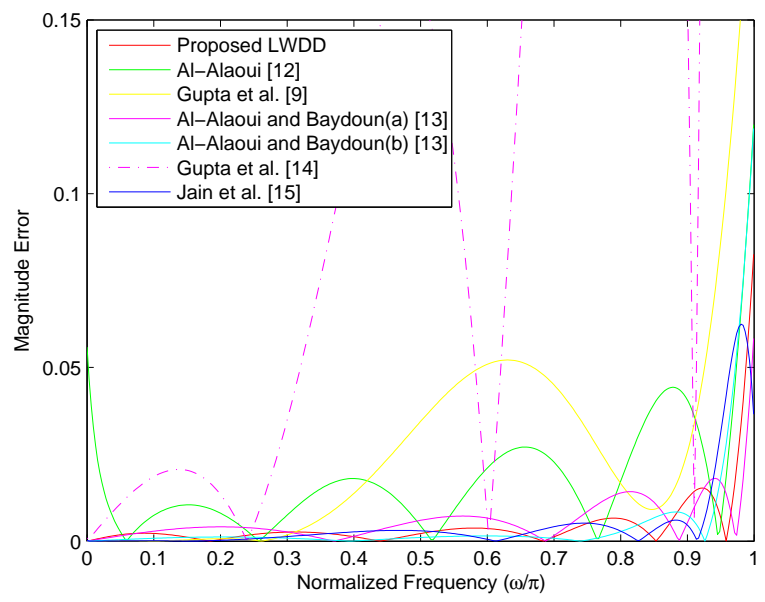

Fig. 9. Magnitude error comparison of the proposed 3rd order LWDD and existing differentiators.

It is discernible that the deviation of magnitude from ideality varies least in case of the proposed LWDD over a wide range of frequency. Figures 10 and 11, represent the graphical comparison of magnitude response and phase response of the 5th order LWDD and the existing 5th order differentiator mentioned in Tab. 3. Further in Fig. 12, the magnitude error plot of the designed and existing 5th order differentiator is shown. All reported figures depict that the proposed minimum multiplier LWDD approximates the magnitude response of the ideal differentiator over wide frequency band in comparison to the reported differentiators.

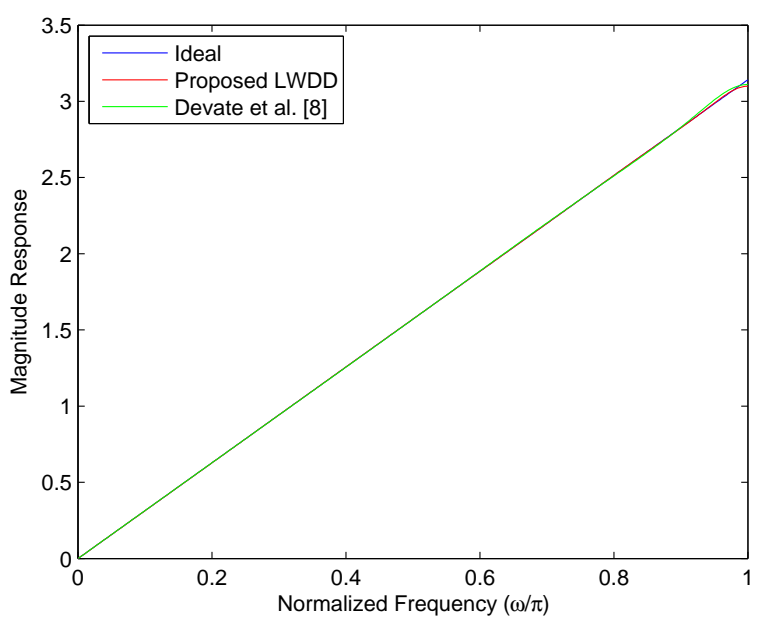

Fig. 10. Magnitude response comparison of the proposed 5 th order LWDD and existing differentiator.

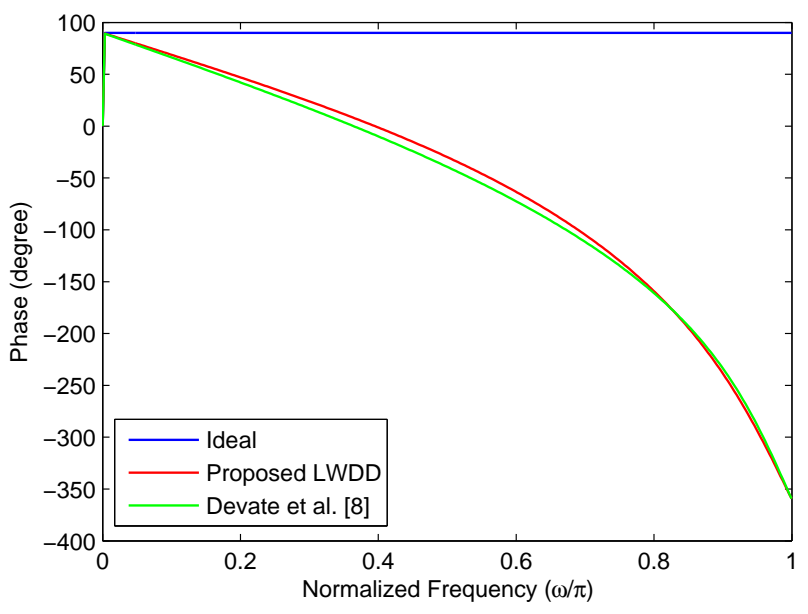

Fig. 11. Phase response comparison of the proposed 5th order LWDD and existing differentiator.

For the affirmative analysis, absolute magnitude error, mean relative error $(\mathrm{dB})$ and number of multiplier operations are the main parameters taken into consideration to evaluate performance of the designed LWDDs. The value of AME, MRE (dB) and MPE of the proposed minimum multiplier 3rd and 5th order LWDD and different reported differentiators are provided in Tab. 4. The absolute magnitude error is observed to be the lowest (1.4706 and 0.3243, respectively) for the proposed 3rd and 5th order LWDD amongst all reported differentiators. The MRE and mean phase error are evaluated over the complete frequency range. The MPE (rad) is calculated using the following equation 


\begin{tabular}{clccc}
\hline Order & Reference & $\begin{array}{c}\text { Absolute magnitude } \\
\text { error }\end{array}$ & $\begin{array}{c}\text { Mean relative } \\
\text { error (dB) }\end{array}$ & $\begin{array}{c}\text { Mean phase } \\
\text { error (rad) }\end{array}$ \\
\hline 3rd & Proposed LWDD & 1.4706 & -51.7828 & 1.4896 \\
& Al-Alaoui [12] & 5.5590 & -13.3765 & 3.7851 \\
& Gupta et al. [9] & 8.4601 & -38.0100 & 0.4117 \\
& Al-Alaoui and Baydoun (a) [13] & 1.8570 & -46.9583 & 0.6726 \\
& Al-Alaoui and Baydoun (b) [13] & 1.5120 & -52.7418 & 0.6682 \\
& Gupta et al. [14] & 91.4645 & -17.8199 & 1.8253 \\
& Jain et al. [15] & 1.5372 & -52.9934 & 0.6632 \\
\hline 5th & Proposed LWDD & 0.3243 & -64.0481 & 2.5895 \\
& Devate et al. [8] & 0.9008 & -57.4759 & 2.6660 \\
\hline
\end{tabular}

Tab. 4. Statistical comparison of the designed LWDD with existing designs.

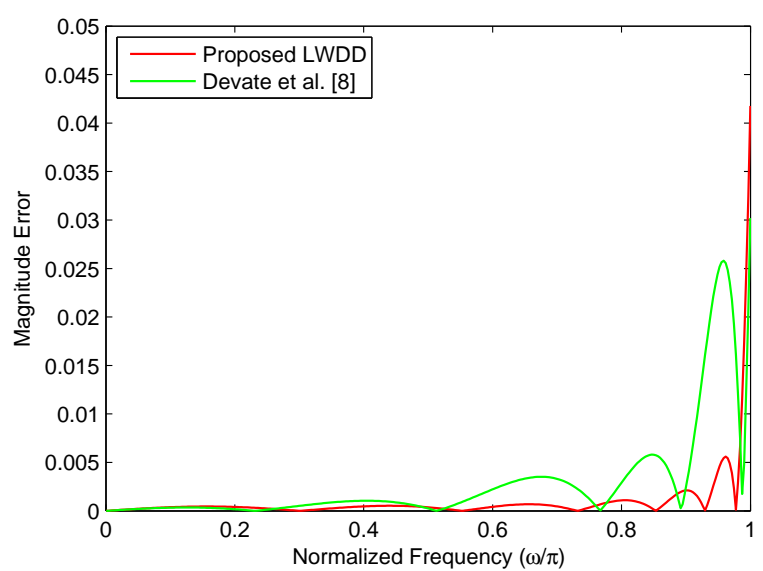

Fig. 12. Magnitude error comparison of the proposed 5th order LWDD and existing differentiator.

$$
M P E=\frac{1}{N} \sum_{i=1}^{N}\left|\frac{\angle H_{\mathrm{d}}(\omega)-\angle H_{\mathrm{LWDF}}(\omega)}{\angle H_{\mathrm{d}}(\omega)}\right|, \quad 0 \leq \omega \leq \pi .
$$

Comparative results reveal that the 3 rd and 5 th order differentiators designed using LWDF possess the lowest MRE (dB) $(-51.7828 \mathrm{~dB}$ and $-64.0481 \mathrm{~dB}$, respectively) amongst all. However, MPE of the proposed 3rd order LWDD is slight higher than that of differentiator designed using GA and MPZCO [13], [15]. Whereas, MPE of the proposed 5 th order LWDD is lower than the differentiator proposed by Devate et al. [8]. On the grounds of the above facts, it is inferred that the proposed minimum multiplier wideband LWDD performs better than the reported differentiators by approaching the ideal curve to the maximum and also inherit all the advantageous properties of LWDF.

Transfer function of a recursive differentiator can be realized using different structures. For infinite precision arithmetic all structures produce the same output for a given input, but under finite precision arithmetic different kinds of errors occur. The best structure is a trade-off between many different aspects such as stability, coefficient sensitivity and other quantization effects. Hence, need of the competent structures for differentiation applications is rectified by using lattice wave digital structural realization. Estimation of hardware requirements of the proposed LWDD structure

\begin{tabular}{clc}
\hline Order & Differentiator & $\begin{array}{c}\text { Number of } \\
\text { Multipliers }\end{array}$ \\
\hline designs & Proposed LWDD & 4 \\
& Al-Alaoui & 7 \\
& Gupta et al. & 5 \\
& Al-Alaoui and Baydoun(a) & 6 \\
& Al-Alaoui and Baydoun(b) & 7 \\
& Gupta et al. & 5 \\
& Jain et al. & 7 \\
\hline 5th & Proposed LWDD & 6 \\
& Devate et al. & 11 \\
\hline
\end{tabular}

Tab. 5. Multiplication operations required by the proposed LWDD and the existing differentiators.

can be done by counting the number of multiplications required for its implementation. The number of multiplication operations required by the proposed 3 rd and 5 th order differentiators are computed from the signal-flow graph provided in Figures 5 and 6, respectively. Arithmetic complexity of the reported digital differentiators is computed by considering their realization using direct form II structure.

The number of multiplication operations required by the proposed LWDD and the existing differentiators are provided in Tab. 5, which are computed from the signal-flow graph. The number of multiplication operations required by the proposed $N$ th order LWDD is $N+1$ multiplication, whereas, the exiting $N$ th order differentiator structures require $2 N+1$ multiplication. The proposed $3 \mathrm{rd}$ and 5 th order LWDD require $43 \%$ and $45 \%$ less multipliers as compared to the reported 3rd and 5th order differentiators. This implies that the LWDD yields a smaller arithmetic complexity, in terms of multiplications, compared to the existing ones leading to minimum hardware cost and making it suitable for VLSI implementation. Based on the observations, it is ratified that the proposed minimum multiplier LWDD outperforms all the existing differentiators both in design as well as structural realization.

\section{Conclusion}

A novel method for designing of minimum multiplier digital differentiator based on lattice wave digital filter and $L_{1}$-CSA is proposed. The optimal coefficients of 3 rd and 5 th 
order lattice wave digital differentiator are procured by minimizing the $L_{1}$-error objective function by means of cuckoo search algorithm. The proposed LWDDs accurately approximate the ideal response with very small AME and MRE over the entire frequency range. Simulation results clearly demonstrate the effective performance of the proposed differentiator over other existing differentiators along with reduced computational complexity. A significant improvement in hardware utilization is achieved by incorporating the lattice wave digital structural realization which makes the proposed LWDD useful in various applications.

\section{References}

[1] AL-ALAOUI, M. A. Novel FIR approximations of IIR differentiators with applications to image edge detection. In Proceedings of the 18th IEEE International Conference on Electronics, Circuits and Systems (ICECS). Beirut (Lebanon), 2011, p. 11-14. DOI: 10.1109/ICECS.2011.6122335

[2] LAGUNA, P., THAKOR, N. V., CAMINAL, P., et al. Low-pass differentiators for biological signals with known spectra: Application to ECG signal processing. IEEE Transactions on bio-medical engineering, 1990, vol. 37, no. 4, p. 420-425. DOI: 10.1109/10.52350

[3] SKOLNIK, M. I. Introduction to Radar Systems. 2nd ed. New York, NY (USA): McGraw \& Hill, 1980. ISBN: 0070579091

[4] XU, Y., DAI, T., SYCARA, K., et al. Service level differentiation in multi-robots control. In Proceedings of the International Conference on Intelligent Robots and Systems (IROS). Taipei (China), 2010, p. 2224-2230. DOI: 10.1109/IROS.2010.5649366

[5] AL-ALAOUI, M. A. Novel digital integrator and differentiator. IET Electronics letters, 1993, vol. 29, no. 4, p. 376-378. DOI: $10.1049 / \mathrm{el}: 19930253$

[6] NGO, N. Q. A new approach for the design of wideband digital integrator and differentiator. IEEE Transactions on Circuits and Systems II: Express Briefs, 2006, vol. 53, no. 9, p. 936-940. DOI: 10.1109/TCSII.2006.881806

[7] GUPTA, M., JAIN, M., KUMAR, B. Novel class of stable wideband recursive digital integrators and differentiators. IET Signal Processing, 2010, vol. 4, no. 5, p. 560-566. DOI: 10.1049/iet-spr.2009.0030

[8] DEVATE, J., KULKARNI, S. Y., PAI, K. R. Wideband IIR digital integrator and differentiator using curve fitting technique. In Proceedings of the International Conference on Signal Processing, Communication and Networking (ICSCN). 2015, p. 1-4. DOI: 10.1109/ICSCN.2015.7219845

[9] GUPTA, M., JAIN, M., KUMAR, B. Recursive wideband digital integrator and differentiator. International Journal of Circuit Theory and Applications, 2011, vol. 39, no. 7, p. 775-782.

[10] UPADHYAY, D. K. Class of recursive wideband digital differentiators and integrators. Radioengineering, 2012, vol. 21, no. 3, p. 904-910. ISSN: 1805-9600

[11] UPADHYAY, D. K. Recursive wideband digital differentiators. IET Electronics letters, 2010, vol. 46, no. 25, p. 1661-1662. DOI: 10.1049/el.2010.2113

[12] AL-ALAOUI, M. A. Class of digital integrators and differentiators. IET Signal Processing, 2011, vol. 5, no. 2, p. 251-260. DOI: $10.1049 /$ iet-spr.2010.0107
[13] AL-ALAOUI, M. A., BAYDOUN, M. Novel wideband digital differentiators and integrators using different optimization techniques. In Proceedings of the International Symposium on Signals, Circuits and Systems (ISSCS). Iasi (Romania), 2013, p. 1-4. DOI: 10.1109/ISSCS.2013.6651225

[14] GUPTA, M., RELAN, B., YADAV, R., et al. Wideband digital integrators and differentiators designed using particle swarm optimisation. IET Signal Processing, 2014, vol. 8, no. 6, p. 668-679. DOI: $10.1049 /$ iet-spr.2013.0011

[15] JAIN, M., GUPTA, M., JAIN, N. K. Analysis and design of digital IIR integrators and differentiators using minimax and pole, zero, and constant optimization methods. ISRN Electronics, 2013, Article ID: 493973. DOI: 10.1155/2013/493973

[16] GUPTA, M., JAIN, M., JAIN, N. Linear phase second order recursive digital integrators and differentiators. Radioengineering, 2012, vol. 21 , no. 2 , p. 712-717. ISSN: $1805-9600$

[17] FETTWEIS, A. Wave digital filters: Theory and practice. IEEE Proceeding, 1986, vol. 74, no. 2, p. 270-327. DOI: 10.1109/PROC.1986.13458

[18] YLI-KAAKINEN, J., SARAMÄKI, T. A systematic algorithm for the design of lattice wave digital filters with short-coefficient wordlength. IEEE Transaction on Circuits and Systems-I, 2007, vol. 54, no. 8, p. 1838-1851. DOI: 10.1109/TCSI.2007.902513

[19] GAZSI, L. Explicit formulas for lattice wave digital filters. IEEE Transaction on Circuits and Systems, 1985, vol. 32, no. 1, p. 68-88. DOI: 10.1109/TCS.1985.1085595

[20] AGGARWAL, M., BARSAINYA, R., RAWAT, T. K. FPGA implementation of Hilbert transformer based on lattice wave digital filters. In Proceedings of the IEEE Conference on Reliability, Infocom Technologies and Optimization (ICRITO). Noida (India), 2015, p. 1-5. DOI: 10.1109/ICRITO.2015.7359331

[21] BARSAINYA, R., AGgARWAL, M., RAWAT, T. K. Multiplierless implementation of quadrature mirror filter. In Proceedings of the IEEE Conference on Reliability, Infocom Technologies and Optimization (ICRITO). Noida (India), 2015, p. 1-6. DOI: 10.1109/ICRITO.2015.7359328

[22] BARSAINYA, R., AGGARWAL, M., RAWAT, T. K. Minimum multiplier implementation of a comb filter using lattice wave digital filter. In Proceedings of the Annual IEEE India Conference (INDICON). New Delhi (India), 2015, p. 1-6. DOI: 10.1109/INDICON.2015.7443491

[23] GROSSMANN, L. D., ELDAR, Y. C. An $L_{1}$-method for the design of linear-phase FIR digital filters. IEEE Transactions on Signal Processing, 2007, vol. 55, no. 11, p. 5253-5266. DOI: 10.1109/TSP.2007.896088

[24] HASHIM, H. A., EL-FERIK, S., ABIDO, M. A. A fuzzy logic feedback filter design tuned with PSO for $L_{1}$ adaptive controller. Expert Systems with Applications, 2015, vol. 42, no. 23, p. 9077-9085. DOI: 10.1016/j.eswa.2015.08.026

[25] YANG, X. S., DEB, S. Cuckoo search via Lévy flights. In Proceedings of the 2009 World Congress on Nature \& Biologically Inspired Computing (NaBIC). Coimbatore (India), 2009, p. 210-214. DOI: 10.1109/NABIC.2009.5393690

[26] YANG, X. S., DEB, S. Engineering optimisation by cuckoo search. International Journal of Mathematical Modelling and Numerical Optimisation, 2010, vol. 1, no. 4, p. 330-343. arXiv: 2010arXiv1005.2908Y

[27] YANG, X. S., DEB, S. Cuckoo search: Recent advances and applications. Neural Computing and Applications, 2014, vol. 24, no. 1, p. 169-174. DOI: $10.1007 / \mathrm{s} 00521-013-1367-1$ 


\section{About the Authors ...}

Richa BARSAINYA incurred her B. Tech degree in Electronics and communication Engineering in the year 2010 from Bundelkhand University, Uttar Pradesh. She completed M. Tech in signal processing from Ambedkar Institute of Technology, Guru Gobind Singh Indraprastha University in 2012. Presently, she is pursuing Ph.D. in the field of wave digital filter design from Netaji Subhas Institute of Technology, University of Delhi.
Tarun Kumar RAWAT received his M. Tech and $\mathrm{Ph}$.D. from NSIT, University of Delhi, in 2003 and 2010, respectively. Presently, he is working in NSIT as an Assistant Professor in the Department of Electronics and Communication Engineering department. His teaching and research work include digital signal processing, statistical signal processing, VLSI signal processing and wave digital filter. He has authored/coauthored over 50 research papers and contributed two books: Signal and System and Digital Signal Processing in Oxford University Press. 